\title{
MÉTODOS ANTICONCEPTIVOS: TASA DE PREVALENCIA Y CARACTERIZACIÓN EN MUJERES DEL EJE CAFETERO, COLOMBIA, 2016-2019
}

\author{
Franklin José Espitia De La Hoz
}

\section{RESUMEN}

Objetivo: Determinar la prevalencia y caracterizar los métodos anticonceptivos en mujeres del Eje Cafetero, y establecer los efectos adversos de los métodos hormonales. Materiales y métodos: Estudio de corte transversal. Se incluyeron 1875 mujeres mayores de 18 años de edad, sexualmente activas y residentes en el Eje Cafetero. La investigación se realizó en la consulta externa de ginecología de seis instituciones hospitalarias (públicas y privadas), en las 6 principales ciudades del Eje Cafetero (Armenia, Calarcá, Pereira, Dos Quebradas, Manizales y Chinchiná); entre julio de 2013 y junio de 2019. Se hizo análisis descriptivo. Se hace descripción estratificada por edad a partir de los 18 años. Resultados: La edad arrojó una media de 26,45 $\pm 3,72$ años. La prevalencia de anticoncepción - planificación familiar en las mujeres del Eje Cafetero es del 78,24 \%. Los métodos hormonales son los más usados $(69,12 \%)$, seguido por la esterilización (19,23 \%). Entre los hormonales, los orales son los más utilizados $(60,05 \%)$ y en segundo lugar los implantes $(24,26 \%)$. La mastalgia es el principal efecto adverso (32,92 \%) seguido por el acné $(27,19 \%)$. La alteración de la función sexual se presentó en el 7,39 $\%$ de las usuarias de métodos hormonales. Conclusiones: cerca de las $3 / 4$ partes de las mujeres del Eje Cafetero utiliza un método anticonceptivo, siendo los métodos hormonales los de elección en las $2 / 3$ de la población, con $1 / 3$ de efectos adversos que no afectan la tasa de satisfacción.

Palabras Clave: Anticonceptivos; Planificación Familiar; Prevalencia; Métodos; Mujeres. (Fuente: DeCS BIREME).

\section{CONTRACEPTIVE METHODS: PREVALENCE RATE AND CHARACTERIZATION IN WOMEN OF THE EJE CAFETERO, COLOMBIA, 2016-2019}

\begin{abstract}
Objective: To determine the prevalence and characterize contraceptive methods in women of the Coffee Region, and to establish the adverse effects of hormonal methods. Materials and methods: Cross-sectional study. 1875 women over 18 years of age, sexually active and residing in the Coffee Axis were included. The research was carried out in the gynecology outpatient clinic of six hospital institutions (public and private), in the 6 main cities of the Coffee Region (Armenia, Calarcá, Pereira, Dos Quebradas, Manizales and Chinchiná); between July 2013 and June 2019. Descriptive analysis was performed. A stratified description is made by age from 18 years. Results: Age showed an average of $26.45 \pm 3.72$ years. The prevalence of contraception - family planning in the women of the Coffee Region is $78.24 \%$. Hormonal methods are the most used $(69.12 \%)$, followed by sterilization (19.23\%). Among the hormonal ones, the oral ones are the most used $(60.05 \%)$ and secondly the implants $(24.26 \%)$. Mastalgia is the main adverse effect $(32.92 \%)$ followed by acne $(27.19 \%)$. Altered sexual function occurred in $7.39 \%$ of users of hormonal methods. Conclusions: about las parts of women in the Coffee Region use a contraceptive method, with hormonal methods being the ones of choice in las of the population, with $1 / 3$ of adverse effects that do not affect the satisfaction rate.
\end{abstract}

Key Words: Contraceptive Agents; Family Planning (Public Health); Prevalence; Methods; Women (Source: MeSH NLM).

\section{INTRODUCCIÓN}

Los métodos anticonceptivos se han usado desde la antigüedad, los cuales surgieron ante la necesidad de decidir el momento de la concepción ${ }^{1}$. Según Espitia et al ${ }^{1}$, en un estudio realizado en 12 ciudades de Colombia, el $89,83 \%$ de las mujeres utilizan métodos anticonceptivos, con predominio de la anticoncepción hormonal (58,99 \%).
Existe una amplia variedad de métodos anticonceptivos, pudiéndose clasificar de diferentes formas y maneras dependiendo de distintas variables: naturales o artificiales, hormonales y no hormonales, quirúrgicos y no quirúrgicos, temporales o definitivos, vaginales y de barrera ${ }^{1-3}$.

En Colombia se calcula que alrededor de 3 millones de mujeres están expuestas a un embarazo no planeado,

Ginecología y Obstetricia / Universidad Militar nueva Granada

Sexología Clínica - Master en sexología: educación y asesoramiento sexual / Universidad de Alcalá de Henares.

Uroginecología / FUCS - Hospital de San José / Unicamp, Brasil.

Director científico: Hathor, Clínica Sexológica.

https://orcid.org/0000-0002-4581-9680

Citar como: Espitia De La Hoz FJ. Métodos anticonceptivos: tasa de prevalencia y caracterización en mujeres del eje cafetero, Colombia, 2016-2019.Rev Peru Investig Matern Perinat 2020; 9(3):10-17

DOI https://doi.org/10.33421/inmp.2020200

Recibido: 13-08-20 Aprobado: 30-09-20 
debido al no uso de un método anticonceptivo o a la utilización inconsistente o inadecuada del mismo ${ }^{1}$.

Los anticonceptivos hormonales, debido a su seguridad y tolerabilidad, gracias a la progresiva disminución de la dosis de estrógeno han logrado, además de disminuir el riesgo de enfermedad trombo-embólica, que efectos adversos frecuentes (náuseas, vómitos, cefalea y mastalgia) sean cada vez menores ${ }^{4,5}$, lo cual los ha convertido en la primera línea de elección para las usuarias sin interés reproductivo en los próximos años. Entre los métodos hormonales se destacan los anticonceptivos orales, combinados (ACO) y de progestágeno, los inyectables, el anillo vaginal, el parche transdérmico, implantes subcutáneos y DIU de levonorgestrel ${ }^{1,6,7}$.

A pesar de la amplia utilización y progresivo mejoramiento de los anticonceptivos, siguen siendo muchos los efectos adversos asociados a su uso, los cuales dependen de diferentes factores (tipo de anticonceptivo, dosificación del estrógeno, enfermedades asociadas, modo de uso, entre otros factores asociados a la usuaria del método) $)^{1,8}$.

Se conoce que en los países donde se han establecido políticas liberales hacia la educación sexual y la disponibilidad de anticonceptivos, estos son utilizados de forma más eficaz, con tasas de embarazos más bajas ${ }^{9}$; sin embargo, los estimativos de la división de población de las Naciones Unidas, es que la población mundial aumentará en un $50 \%$, desde 6.100 millones de personas (a mediados del 2001) hasta 9.300 millones en el año $2050^{10}$.

Es importante, a nivel regional, conocer el comportamiento poblacional acerca del uso de los métodos anticonceptivos, así como de sus efectos adversos, para poder definir si estamos cumpliendo con las recomendaciones nacionales e internacionales; a fin de planear acciones de mejoramiento y de prevención de la deserción o abandono de la anticoncepción - planificación responsable. De este modo, el presente estudio tiene como objetivo principal determinar la prevalencia y caracterizar los métodos anticonceptivos $y$, secundariamente, establecer los efectos adversos de los métodos hormonales en mujeres del Eje Cafetero.

\section{MATERIALES Y MÉTODOS}

Diseño y población: Estudio observacional descriptivo de corte transversal, en una muestra de 1.875 participantes. Se incluyeron mujeres mayores de 18 años de edad, sexualmente activas y residentes en el Eje Cafetero. Se excluyeron las mujeres con diagnóstico de cáncer, antecedente personal de disfunción sexual y las que no aceptaron participar en el estudio; entre el 01 de julio de 2013 y el 30 de junio de 2019. La investigación se realizó en la consulta externa de ginecología de seis instituciones hospitalarias (públicas y privadas), en las 6 principales ciudades del Eje Cafetero. Estas son de carácter universitario, atienden a personas pertenecientes a los regímenes de aseguramiento contributivo y subsidiado por el Estado en el Sistema de seguridad social en Colombia. El Eje Cafetero es una región de Colombia, ubicada en los departamentos de Caldas, Risaralda y Quindío; cuyas ciudades capitales son Manizales, Pereira y Armenia, respectivamente). Se localiza en el centrooccidente de Colombia, haciendo parte de la Región Andina (cordilleras Occidental y Central). La población femenina es mayoritaria con el $52,96 \%$ del total ${ }^{11}$. Se calculó una muestra de 1067 participantes, con base en la población de mujeres, con un intervalo de confianza del $95 \%$, y un error de $3 \%$. Se aplicó muestreo aleatorio simple, mediante un programa de computadora, para selección de las mujeres.

Procedimiento: A cada mujer se le realizó una entrevista personalizada, inicialmente por parte del especialista, luego por el personal de enfermería, entrenado en el proceso de captación de pacientes y diligenciamiento de formularios. Se hizo seguimiento cada seis meses hasta finalizar el estudio. Las mujeres que cumplían con los criterios de selección y aceptaron participar, se les explicaban los objetivos de la investigación.

Se realizó una valoración complementaria donde se finalizó el interrogatorio dirigido a los hábitos, antecedentes médicos personales y se completaron los socio-demográficos y culturales. A su vez se diligenció el instrumento "Índice de Función Sexual Femenina (IFSF)". Los datos fueron consignados en una tabla de Excel 2007 (v12.0).

Variables medidas: socio-demográficas (edad, raza, nivel de estudios, estrato socio-económico, estado civil, ocupación, condición espiritual o religiosa, área de residencia, afiliación al sistema general de seguridad social en salud); talla, peso, Índice de masa corporal (IMC); hábitos (ingesta de alcohol, tabaquismo, consumo de café, sedentarismo, adicción a sustancias psicoactivas); variables de salud sexual y reproductiva (edad de la menarquia, tipo de método anticonceptivo y afectos adversos, antecedente de abuso sexual o violencia sexual en el matrimonio, tiempo de convivencia en pareja); variables de comportamiento sexual: edad de inicio de la actividad sexual coital, actividad sexual (masturbación, sexo oral, coito -vaginal o anal-), frecuencia promedio de relaciones sexuales coitales semanales, número de parejas sexuales. Se indagaron además las preguntas de los dominios del instrumento IFSF.

El "Índice de Función Sexual Femenina (IFSF)" está validado en español ${ }^{12}$. Este cuestionario ha sido ampliamente aplicado en la población colombiana para caracterizar la función sexual femenina ${ }^{11,13}$. Lo conforman 19 ítems agrupados en seis dominios: deseo, excitación, lubricación, orgasmo, satisfacción y dolor. Cada pregunta tiene 5 ó 6 alternativas de respuesta, con una puntuación 
que varía entre 0 a 5; cuya puntuación final total oscila entre 2 y 36 , obtenida mediante la suma aritmética de los productos obtenidos al multiplicar el promedio de cada dimensión por un factor. A mayor puntaje, mejor función sexual. Una puntuación $\leq 26,55$ establece la diferencia entre las mujeres con y sin riesgo de disfunción sexual, o si alguna de las dimensiones es menor a 3,6 $6^{11-13}$.

Análisis estadístico: Las variables cualitativas se expresaron como frecuencias absolutas y relativas (porcentajes), y las cuantitativas como mediana, media y desviación estándar y su rango. Se hizo un análisis descriptivo de todas las variables. Se calculó odds ratios (OR) y los intervalos de confianza de 95\% (IC95\%). El nivel de significación se estableció con $p<0,05$. La tasa de prevalencia de anticoncepción - planificación familiar, se obtuvo del número total de participantes/total de mujeres que utilizaban algún método anticonceptivo. Los datos se analizaron con el programa estadístico IMB SPSS Statistics 20.0 .

Aspectos éticos: El estudio fue aprobado por el Comité de Ética institucional. Se siguieron los principios éticos de la declaración de Helsinki y la Resolución 8430 de 1993 del ministerio de Salud de Colombia. Se garantizó la confidencialidad de la información de las pacientes. Los principios de confidencialidad y privacidad, para todas las mujeres incluidas, se garantizaron y preservaron.

\section{RESULTADOS}

Una población total de 2357 mujeres fue seleccionada a participar en la investigación; de estas, 169 (7,17 \%) se negaron a participar. A las 2.188 mujeres restantes se les solicitó diligenciar el IFSF, pero un total de $103(4,7 \%)$ se retiraron de manera voluntaria por incomodidad ante algunas preguntas de las variables relacionadas con su salud sexual y reproductiva, quedando 2085 encuestas, de estas se encontraron $79(3,78 \%)$ con fallas en el diligenciamiento, por lo que se excluyeron. Se mudaron de la región $29(1,32 \%)$, se perdieron porque no volvieron o cambiaron de teléfono 35 (1,59\%), abandonaron la anticoncepción por interés reproductivo $41(1,87 \%)$ y se cambiaron de centro de atención $26(1,18 \%)$. De esta manera, para el análisis final se tomó en cuenta un total de 1875 (85,69\%) participantes.

La edad de la población total arrojó una media de 26,45 $\pm 3,72$ años, mediana 33 años, rango entre 18 y 51 . Las principales características socio-demográficas reportan que, de las 1875 mujeres participantes, el 51,84\% eran mayores de 30 años, el 21,44 \% tenían bajo nivel de escolaridad, el 48,96 \% eran amas de casa, el 76,16 $\%$ estaban casadas o vivían en unión libre. El 71,84 \% pertenecían al régimen contributivo de seguridad social en salud, y el 83,84\% residían en el área urbana. El 86,56\% profesaban la religión católica. En la tabla 1 se describen detalladamente las características socio-demográficas.
Tabla 1. Características socio-demográficas de las mujeres usuarias de métodos anticonceptivos en el Eje Cafetero, Colombia, 2016-2019

\begin{tabular}{|c|c|}
\hline Variable y categorías & \\
\hline Edad: $X \pm D E$ años & $26,45 \pm 3,72$ \\
\hline Edad de la pareja: $X \pm D E$ años & $29,76 \pm 4,59$ \\
\hline Peso: $\mathrm{X} \pm \mathrm{DE} \mathrm{Kg}$ & $61,23 \pm 8,46$ \\
\hline Talla: $\mathrm{X} \pm \mathrm{DE} \mathrm{Cms}$ & $157,29 \pm 5,73$ \\
\hline IMC: $X \pm D E$ & $24,72 \pm 8,19$ \\
\hline \multicolumn{2}{|l|}{ Raza } \\
\hline Hispánicas & $837(55,05 \%)$ \\
\hline Indígenas & $15(1,02 \%)$ \\
\hline Afrocolombianas & $615(41,92 \%)$ \\
\hline \multicolumn{2}{|l|}{ Estrato socio-económico } \\
\hline Alto & $177(12,06 \%)$ \\
\hline Medio & $708(48,26 \%)$ \\
\hline Bajo & $582(39,67 \%)$ \\
\hline \multicolumn{2}{|l|}{ Nivel de estudios } \\
\hline Primaria & $120(8,17 \%)$ \\
\hline Secundaria & $309(21,06 \%)$ \\
\hline Técnicos & $441(30,06 \%)$ \\
\hline Profesionales & $597(40,69 \%)$ \\
\hline
\end{tabular}

La edad de la menarquia fue de 12,75 $\pm 1,89$ años, mediana 11 años, rango entre 9 y 15 . Se encontró que el porcentaje de mujeres usuarias de un método anticonceptivo durante los últimos doce meses, al ingreso del estudio, fue de $37,42 \%$ (IC95\% 30,12\% - 39,24\%).

La edad de inicio de la actividad sexual arrojó un promedio de 16,59 $\pm 2,24$ años, mediana de 17 años, rango entre 9 y 21. La práctica sexual más frecuente es el coito vaginal (100\%), y la menos frecuente el coito anal $(30,24 \%)$; la masturbación es considerada una práctica común $(86,88$ $\%)$. A la pregunta ¿cuántas veces tuvo relaciones sexuales la semana pasada? (periodo definido como el lapso de los siete días anteriores), el 69,28 \% informó tener relaciones tres veces por semana (rango entre 0 y 12).

Los antecedentes obstétricos reportaron una mediana de 3 hijos en la paridad (rango entre 0 y 6 hijos). Un total de $1.437(76,64 \%)$ mujeres informaron haber tenido más de un embarazo, de los cuales $1.209(84,13 \%)$ fueron no planeados, y de estos $12,15 \%$ se consideraron no deseados; alcanzándose un 5,95 \% (72/1.209) de interrupción voluntaria del embarazo (IVE). El 10,08\% eran nulíparas.

Respecto a los hábitos, el 14,88 \% eran fumadoras habituales, el 90,56\% consumían alcohol (una a dos veces por semana) y el 7,52 \% usaban sustancias psicoactivas, el consumo de café se detectó en el 78,88\%. El 42,56 \% de las mujeres participantes se declararon sedentarias.

En cuanto a los comportamientos sexuales, el número de parejas sexuales reportó una mediana de 9 (rango entre $1 \mathrm{y} \geq 24$ ). El $28,64 \%$ refirió más de 5 años de tiempo de convivencia en pareja (rango 1 y 12 años). El 8,96\% afirmó ser bisexual y el 6,24\% homosexual. El 3,68\% 
expresó ser infiel, mientras que el 9,92 \% refirió haberlo sido por lo menos en una ocasión. El 25,76 \% afirmó que la pareja le había sido infiel.

El abuso sexual, en algún momento de la vida, en la población total reportó un 4,96 \% ( $\mathrm{n}=93 / 1.875)$, mientras que la violencia sexual, dentro del matrimonio, (agresión sexual perpetrada por la pareja) registró 12,32 \% $(n=231 / 1875)$.

La tasa de prevalencia de anticoncepción - planificación familiar fue de 78,24 \% ( $n=1467 / 1875)$, mientras que el $21,76 \% \quad(n=408 / 1875)$ no utilizan ningún método anticonceptivo. Se observó que el uso de un anticonceptivo, después de un parto o aborto, alcanzó el $74,23 \%$. En cuanto a los métodos anticonceptivos utilizados, los hormonales son los más usados $(69,12 \%$, $\mathrm{n}=1014 / 1875$ ), seguido por la esterilización (Cirugía de Pomeroy) $(19,23 \%, n=195 / 1875)$; y los menos usados

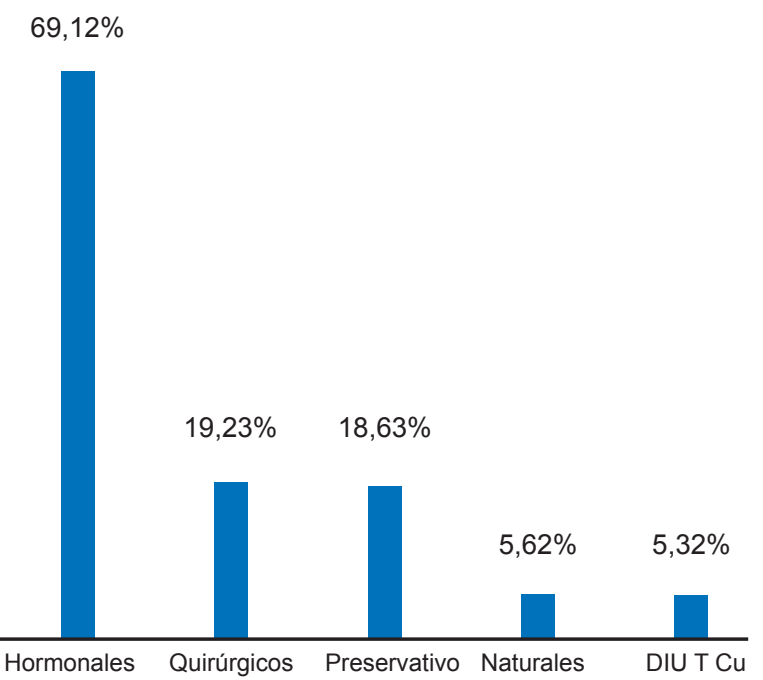

Figura 1. Distribución de los métodos anticonceptivos utilizados por las mujeres en el Eje Cafetero, Colombia, 2016-2019

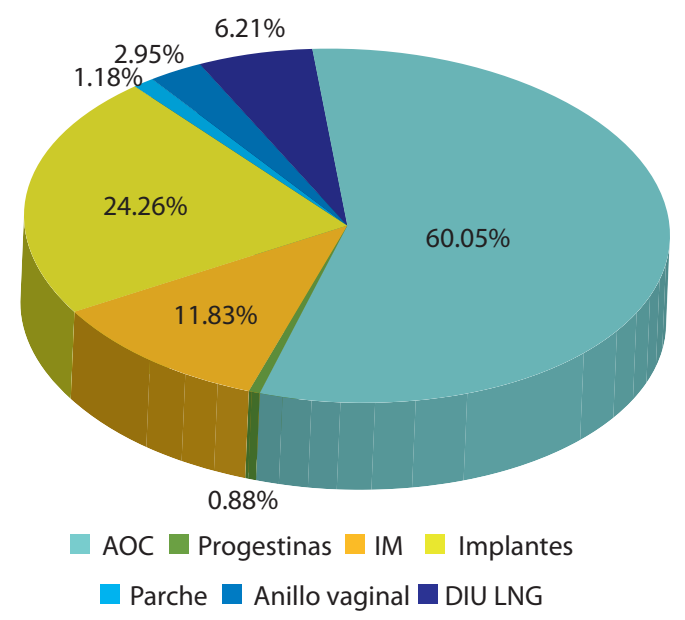

Gráfica 1. Distribución de los anticonceptivos hormonales utilizados por las mujeres en el Eje Cafetero, Colombia, 2016-2019 son los métodos naturales $(5,62 \%, n=57 / 1875)$ (figura 1). El tiempo de uso del método anticonceptivo en el 48,94\% de las usuarias era de más de 6 meses, 22,83\% entre 6 y 12 meses y $30,74 \%$ más de 12 meses. En la gráfica 1 se describe el porcentaje de distribución de los diferentes tipos de métodos hormonales utilizados, mientras que en la tabla 2 se detalla el anticonceptivo hormonal utilizado por las mujeres.

Tabla 2. Tipo de anticonceptivo hormonal utilizado por mujeres del Eje Cafetero, Colombia, 2016-2019

\begin{tabular}{lcc}
\hline \multicolumn{1}{c}{$\begin{array}{c}\text { Nombre del } \\
\text { anticonceptivo }\end{array}$} & Concentración & $\begin{array}{c}\text { Frecuencia } \\
\mathbf{n}(\%)\end{array}$ \\
\hline $\begin{array}{l}\text { Acetato de } \\
\text { medroxiprogesterona }\end{array}$ & $150 \mathrm{mg} / 3 \mathrm{ml}$ & $18(1,77 \%)$ \\
\hline Ciproterona / Etinilestradiol & $2 \mathrm{mg} / 0,035 \mathrm{mg}$ & $14(1,67 \%)$ \\
\hline Clormadinona / Etinilestradiol & $2 \mathrm{mg} / 0,03 \mathrm{mg}$ & $23(2,26 \%)$ \\
\hline Desogestrel & $75 \mathrm{ug}$ & $9(0,88 \%)$ \\
\hline Dienogest / Etinilestradiol & $2 \mathrm{mg} / 0,03 \mathrm{mg}$ & $54(5,32 \%)$ \\
\hline Dienogest / Valerato de estradiol & $132(13,01 \%)$ \\
\hline Drospirenona / Etinilestradiol & $3 \mathrm{mg} / 0,03 \mathrm{mg}$ & $144(14,2 \%)$ \\
\hline $\begin{array}{l}\text { Etonogestrel (implante) } \\
\text { Etonogestrel / Etinilestradiol } \\
\text { (anillo vagina/) }\end{array}$ & $11,7 \mathrm{mg} / 2,7 \mathrm{mg}$ & $30(2,95 \%)$ \\
\hline $\begin{array}{l}\text { Gestodeno / Etinilestradiol } \\
\text { Levonorgestrel (DIU) }\end{array}$ & $75 \mathrm{ug} / 20 \mathrm{ug}$ & $14(1,38 \%)$ \\
\hline $\begin{array}{l}\text { Levonorgestrel (DIU) } \\
\text { Levonorgestrel (implante) }\end{array}$ & $13,5 \mathrm{mg}$ & $24(2,36 \%)$ \\
\hline $\begin{array}{l}\text { Levonorgestrel / } \\
\text { Etinilestradiol }\end{array}$ & $2 \times 75 \mathrm{mg}$ & $39(3,84 \%)$ \\
\hline $\begin{array}{l}\text { Medroxiprogesterona / } \\
\text { Cipionato de estradiol }\end{array}$ & $25 \mathrm{mg} / 0,5 \mathrm{ml} 5$ & $129(12,72 \%)$ \\
\hline $\begin{array}{l}\text { Norelgestromina / } \\
\text { Etinilestradiol (parche) }\end{array}$ & $6 \mathrm{mg} / 0,5 \mathrm{mg} / 600 \mathrm{ug}$ & $12(1,18 \%)$ \\
\hline $\begin{array}{l}\text { Noretisterona / Valerato } \\
\text { estradiol }\end{array}$ & $50 \mathrm{mg} / 5 \mathrm{mg} / 1 \mathrm{cc}$ & $57(5,62 \%)$ \\
\hline & & $150(14,79 \%)$ \\
\hline
\end{tabular}

En cuanto a la distribución por edades, así como del uso de anticonceptivos, se encuentra menor población en las mujeres mayores de 50 años $(3,88 \%)$, con una prevalencia de $15,78 \%$ de uso de anticonceptivos hormonales (tabla 3).

Tabla 3. Distribución por edades y anticonceptivos utilizados por mujeres del Eje Cafetero, Colombia, 20162019

\begin{tabular}{cccc}
\hline $\begin{array}{c}\text { Edad } \\
\text { (años) }\end{array}$ & $\begin{array}{c}\text { Frecuencia } \\
\mathbf{n}(\%)\end{array}$ & $\begin{array}{c}\text { Método } \\
\text { anticonceptivo }\end{array}$ & $\begin{array}{c}\text { Frecuencia } \\
\mathbf{n}(\%)\end{array}$ \\
\hline $18-29$ & $836(56,98 \%)$ & $\begin{array}{c}\text { Hormonal } \\
\text { No hormonal }\end{array}$ & $\begin{array}{c}704(84,21 \%) \\
132(15,78 \%)\end{array}$ \\
\multirow{2}{*}{$30-39$} & $425(28,97 \%)$ & $\begin{array}{c}\text { Hormonal } \\
\text { No hormonal }\end{array}$ & $\begin{array}{c}239(56,23 \%) \\
186(43,76 \%)\end{array}$ \\
\hline \multirow{2}{*}{$40-49$} & \multirow{2}{*}{$149(10,15 \%)$} & Hormonal & $62(41,61 \%)$ \\
& \multirow{2}{*}{50} & No hormonal & $87(58,38 \%)$ \\
\hline
\end{tabular}


La selección del método anticonceptivo usado, en el $89,16 \%$ de las mujeres, fue recomendado por un médico, mientras que el 5,65\% lo hizo gracias al consejo de una amiga, el 3,34 \% por el dependiente de la farmacia, el resto siguió las campañas publicitarias en televisión. Se identificó una satisfacción del 82,64 \% $(n=838 / 1.014)$ con el uso de métodos anticonceptivos hormonales.

Entre los métodos hormonales el más utilizado fue levonorgestrel $0,15 \mathrm{mg} /$ etinilestradiol $0,03 \mathrm{mg}(14,79$ $\%$ ), seguida por drospirenona $3 \mathrm{mg} /$ etinilestradiol 0,03 $\mathrm{mg}(14,2 \%)$, el menos usado fue el desogestrel 75 microgramos $(0,88 \%)$ (figura 2$)$.

Los efectos adversos más referidos fueron la tensión mamaria $(32,92 \%)$ seguida por el acné $(27,19 \%)$. La alteración de la función sexual se presentó en el 7,36 \% de las usuarias de métodos hormonales (tabla 4).

Tabla 4. Efectos adversos relacionados con el uso de anticonceptivos hormonales en el Eje Cafetero, Colombia, 2016-2019

\begin{tabular}{lc}
\hline \multicolumn{1}{c}{ Efectos adversos } & Frecuencia $\mathbf{n}(\%)$ \\
\hline Acné & $399(27,19 \%)$ \\
\hline Afectación del estado de ánimo & $260(17,72 \%)$ \\
\hline Alteración de la función sexual & $108(7,36 \%)$ \\
\hline Amenorrea & $207(14,11 \%)$ \\
\hline Aumento de peso & $276(18,81 \%)$ \\
Cefalea & $193(13,15 \%)$ \\
Cloasma & $78(5,31 \%)$ \\
Mareos & $213(14,51 \%)$ \\
Nauseas & $171(11,65 \%)$ \\
Spotting & $225(15,33 \%)$ \\
Tensión mamaria & $483(32,92)$ \\
Várices & $21(1,43 \%)$ \\
\hline
\end{tabular}

La asociación causal de efectos adversos entre usuarias de los anticonceptivos hormonales orales combinados versus inyectables, no presentaron diferencia significativa (OR = 1,14; IC95\%: 0,72-3,84). El análisis de subgrupos demostró que hay diferencias en los efectos adversos entre las usuarias de anticonceptivos hormonales orales combinados frente a las distintas presentaciones hormonales (implante: OR = 1,35; IC 95\%: 1.08-1,68; DIU LNG: 0,75; IC 95\%: 0,48-0,87; anillo vaginal: 0,81; IC 95\%: 0,66-1,05).

En relación con los efectos adversos sexuales, no se logró determinar si existía un papel protector entre los anticonceptivos de solo progestinas o DIU LNG (OR = 0,42; IC 95\%: 0,18-1,17 y OR = 0,81; IC 95\%: 0,63-1,05).

Los efectos adversos fueron más frecuentes en el grupo etario de 18 a 29 años, predominando los trastornos gastrointestinales (epigastralgia y náuseas) e incremento de peso; seguido del grupo de $\geq 35$ años, en el cual predominaron la tensión mamaria y alteración del estado de ánimo. El 35,78 \% presentó dos efectos adversos, el $29,85 \%$ tres y el $17,17 \%$ cuatro o más, con una mediana de 3 efectos adversos por mujer (rango entre 1 y 6 ).

La puntuación del IFSF en la población total de las 1875 participantes, al inicio del estudio, fue de 31,59 $\pm 5,46$ puntos, con la menor de 19,89 y la mayor de 34,26 puntos, mientras que en las 1467 mujeres que utilizaban algún método anticonceptivo fue de $30,66 \pm 8,83$ puntos (la menor de 18,93 y la mayor de 33,99 puntos); siendo de 29,14 \pm 6,25 puntos (menor de 18,93 y mayor de 31,72 puntos) en la población de usuarias de métodos hormonales. Al final del estudio la puntuación fue de $27,93 \pm 8,28$ puntos (la menor de 17,49 y la mayor de 30,69 puntos) entre las que usaban métodos anticonceptivos hormonales (Tabla 5).

Tabla 5. Índice de función sexual femenina en mujeres usuarias de anticonceptivos hormonales en el Eje Cafetero, Colombia, 2016-2019

\begin{tabular}{lccc}
\hline Dominios & Inicio & Final & $\boldsymbol{p}$ \\
\hline Deseo & $4,47 \pm 1,02$ & $4,17 \pm 1,05$ & 0,024 \\
Excitación & $5,31 \pm 1,53$ & $4,89 \pm 1,47$ & 0,318 \\
Lubricación & $5,28 \pm 1,71$ & $4,77 \pm 1,44$ & 0,372 \\
Orgasmo & $5,43 \pm 1,38$ & $4,71 \pm 1,08$ & 0,714 \\
Satisfacción & $5,19 \pm 1,56$ & $4,86 \pm 1,59$ & 0,349 \\
Dolor & $4,98 \pm 1,63$ & $4,53 \pm 1,65$ & 0,336 \\
Total & $30,66 \pm 8,83$ & $27,93 \pm 8,28$ & 0,018 \\
\hline
\end{tabular}

La prevalencia de disfunción sexual alcanzó el 7,36 \%. El trastorno sexual con mayor frecuencia fue el bajo deseo $(5,11 \%)$, seguido por alteración de la excitación / lubricación caracterizando la sequedad vaginal $(3,88 \%) \mathrm{y}$, en tercer lugar, la alteración del orgasmo (1,84 \%) (figura 2). El $8,79 \%$ presentó dos trastornos sexuales, el $9,61 \%$ tres o más, con una mediana de 2 disfunciones sexuales por mujer (rango entre 0 y 3 ).

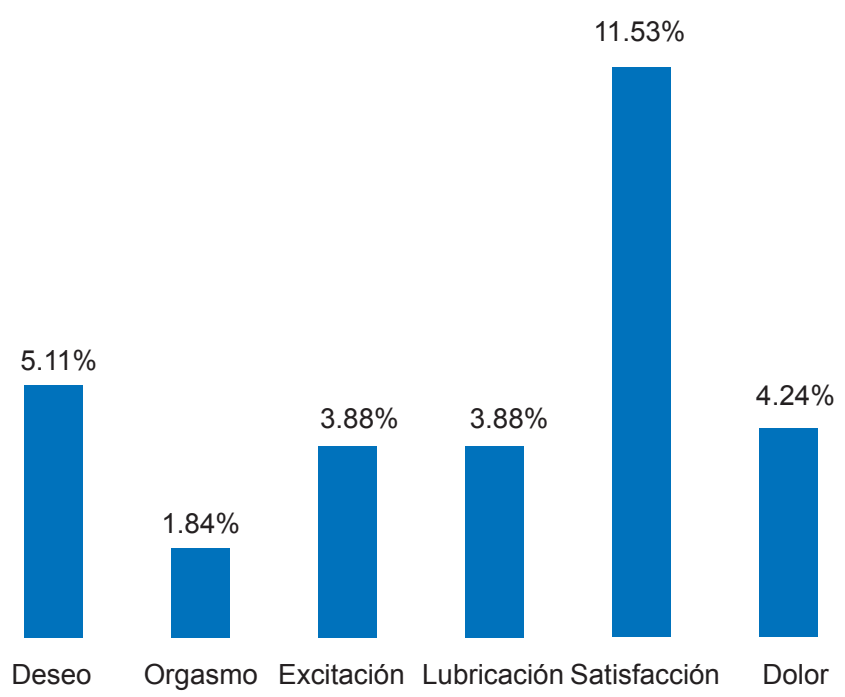

Figura 2. Alteración de la función sexual en mujeres que utilizan anticonceptivos hormonales en el Eje Cafetero, Colombia, 20162019 


\section{DISCUSIÓN}

Los beneficios reportados por el uso de los métodos anticonceptivos van más allá de la planificación familiar; es por eso que también se incluyen entre sus ventajas la prevención de embarazos no planeados, protección contra infecciones de transmisión sexual, regular el ciclo menstrual, efectos dermatológicos, entre otros ${ }^{1,14,15}$.

Los hallazgos de nuestra investigación muestran una significativa prevalencia de uso de métodos anticonceptivos en las mujeres del Eje Cafetero, con un $78,24 \%$, ubicándonos entre las prevalencias de anticoncepción más alta en países latinoamericanos, tales como República Dominicana ${ }^{16}$ con el $71,8 \%$ o Brasil $(79 \%)^{17}$; e incluso comparable a la prevalencia en los Estados Unidos $(75,1 \%)^{18}$, y España $(78,8 \%)^{19}$; aunque superados, según lo informado por la OMS/UNFPA en $2009^{20}$, por países como Noruega $(88,4 \%)$, Reino Unido (82\%) y Francia (81,8\%); aunque muy superior al $60,3 \%$ reportado por Cuervo et $a^{21}$ en la ciudad de Medellín en una muestra de 469 mujeres.

En la presente investigación, entre los métodos más utilizados están los hormonales $(69,12 \%)$ y el preservativo $(17,99 \%)$, siendo los orales los preferidos $(60,02 \%)$ y el implante subdérmico $(24,26 \%)$. En cuanto a nuestros resultados sobre la alta utilización de los anticonceptivos hormonales, observamos que están en concordancia a lo publicado por Gebremedhin et $a^{22}$ en Etiopía, los cuales en una población de 602 mujeres encontraron que el $72,3 \%$ preferían los anticonceptivos inyectables; no obstante, un estudio realizado en Tanzania ${ }^{23}$, en una población de 271 mujeres en edad reproductiva (1549 años), reveló que los anticonceptivos orales eran el método conocido $(81,2 \%)$.

Respecto al anticonceptivo utilizado, nuestros hallazgos, con relación al 5,32 \% de uso del DIU ( $\mathrm{T} \mathrm{Cu}$ ), son similares a los informados por las Naciones Unidas ${ }^{18}$, para América Latina y el Caribe, ya que esta informa que el $6,4 \%$ de las mujeres usan un dispositivo intrauterino ( $7,7 \%$ en el Caribe, $9,5 \%$ en América Central y $4,8 \%$ en América del Sur).

La edad media de población participante es similar a la reportada en estudios españoles $(27,9 \pm 5,9)(24)$ y 28,0 $\pm 6,4^{25}$. Lo cual demuestra que la adecuada información acerca de los diferentes métodos de anticoncepción, facilitan su elección entre las usuarias ${ }^{26}$.

Los hallazgos de los efectos adversos más frecuentes, en nuestra investigación, concuerdan con los publicados por Grossman et $a^{27}$ y Loder et $a^{28}$, aunque nuestros porcentajes son superiores, lo cual puede estar asociado al componente socio-cultural de la población participante.

Nuestros hallazgos sobre los resultados de los efectos negativos en la función sexual, se alinean con Sabatini et $a l^{29}$ y Espitia et $a l^{30}$, los cuales, en su publicación, al hacer el análisis de los eventos adversos, revelaron como punto crucial para la aceptabilidad, el cumplimiento y la continuación de los métodos anticonceptivos hormonales, su influencia en la alteración de las relaciones sexuales debido a la presencia de sequedad vaginal y pérdida del deseo sexual. Por el contrario, Schaffir et $a^{\beta 1}$ en un análisis prospectivo no encontraron cambios en la función sexual; de igual manera Berenson et $a /^{32}$ no reportaron alteraciones en la función sexual, y las mujeres no informaron modificaciones en el deseo sexual en comparación con las que recibieron placebo.

Es incuestionable que el uso de métodos anticonceptivos representa un cambio en el paradigma hacia la autonomía reproductiva de las mujeres, lo que ha impulsado inmensos retos en el empoderamiento femenino, hasta lograr el reconocimiento de los derechos sexuales y reproductivos $^{30,33}$; no obstante, las usuarias deben ser informadas tanto de los efectos adversos, como de los beneficios no anticonceptivos.

Es debatida, pero no rechazada, la influencia de los métodos anticonceptivos hormonales sobre la función sexual de la mujer. En múltiples publicaciones se han observado los efectos adversos sexuales en varios subgrupos de mujeres usuarias de anticoncepción hormonal ${ }^{29,30}$; sin embargo, tampoco se puede desconocer que no existe un patrón de efecto consistente donde se sugiera un determinante hormonal o biológico relacionado con la alteración de la función sexual; ya que los efectos sobre el deseo sexual de la mujer, representan una compleja combinación multifactorial e idiosincrásica, involucrando aspectos biológicos, psicológicos y socioculturales ${ }^{1,34}$.

En el caso de que una mujer presente efectos negativos sobre su función sexual, los cuales puedan estar asociados al uso de métodos anticonceptivos hormonales, y que persistan por más de tres meses, la recomendación es cambiar dicho anticonceptivo; sin embargo, es poca la evidencia para sugerir un método sobre otro. A sabiendas que la función sexual depende de múltiples factores, es útil considerar otras causas de disfunción sexual antes de cambiar el método anticonceptivo, siendo importante evaluar la función sexual, de cada mujer, previo al inicio de la anticoncepción.

Sobresalen como fortalezas de nuestro estudio que los hallazgos son consistentes con investigaciones realizadas en otros lugares del mundo ${ }^{27-29}$. Además de suministrar información relevante para facilitar el abordaje de las estrategias de anticoncepción - planificación familiar en la región del Eje Cafetero. Dentro de las limitaciones están que se centra mayoritariamente en población urbana, lo cual puede generar sesgos de selección a la hora de compararlo con la población rural, sitio donde las expectativas de los métodos anticonceptivos pueden tener una connotación diferente. 
Se requieren más investigaciones, a fin de identificar qué factores pueden tener el mayor efecto sobre la función sexual de la mujer, a la hora de utilizar un método anticonceptivo hormonal.

Finalizamos informado que la educación de la usuaria, puede disminuir las posibilidades de eventuales efectos adversos de los anticonceptivos hormonales, de ahí la importancia de una adecuada asesoría en la práctica clínica.

En conclusión, la prevalencia anticoncepción Planificación familiar, encontrada en las mujeres del Eje Cafetero sobrepasa las $3 / 4$ de la población; con una tasa de satisfacción elevada, a pesar de la presencia de 1/3 de efectos adversos. Se hace imperativa la búsqueda e implementación de estrategias de promoción de los métodos anticonceptivos a todo nivel poblacional, a fin de masificar el uso de los mismos, tanto por sus beneficios anticonceptivos como no anticonceptivos.

Agradecimientos Nuestros agradecimientos a las mujeres participantes en este estudio, así como a las instituciones que facilitaron sus recursos. Asimismo, le expreso mi sincero agradecimiento a Hathor, Clínica Sexológica, centro pionero de la investigación en el Eje Cafetero (Colombia).

Financiamiento: La presente investigación fue autofinanciada por el autor.

Conflicto de intereses: El autor declara no tener ningún conflicto de interés.

\section{REFERENCIAS BIBLIOGRÁFICAS}

1. Espitia-De La Hoz FJ. Educación y asesoramiento sexual para hombres y mujeres. Bogotá. Editorial Nomos S.A. Primera edición. 2018; 8(1):99-100.

2. Espitia-De La Hoz FJ. Prevalence and characterisation of sexual dysfunctions in women, in 12 Colombian cities, 20092016. Rev Colomb Obstet Ginec. 2018; 69(1):9-21. DOI: http://dx.doi.org /10.18597/rcog.3035

3. Jacobstein R, Curtis C, Spieler J, Radloff S. Meeting the need for modern contraception: effective solutions to a pressing global challenge. Int J Gynaecol Obstet. 2013; 121 Suppl 1:S9-15. doi: 10.1016/j.ijgo.2013.02.005

4. Keam SJ, Wagstaff AJ. Ethinylestradiol/drospirenone: a review of its use as an oral contraceptive. Treat Endocrinol. 2003; 2(1):49-70. DOI: 10.2165/00024677-200302010-00005

5. Steiner MJ, Dalebout S, Condon S, Dominik R, Trussell J. Understanding risk: a randomized controlled trial of communicating contraceptive effectiveness. Obstet Gynecol. 2003; 102(4):709-17. DOI: 10.1016/s0029-7844(03)00662-8

6. Trussell J. Contraceptive efficacy. In: Hatcher RA, Trussell J, Stewart F, eds. Contraceptive technology (18th revised edition). New York: Ardent Media, 2004: 773-845. DOI 10.3843/ GLOWM.10375

7. A double-blind study comparing the contraceptive efficacy, acceptability and safety of two progestogen-only pills containing desogestrel 75 micrograms/day or levonorgestrel 30 micrograms/day. Collaborative Study Group on the Desogestrel-containing Progestogen-only Pill. Eur J Contracept Reprod Health Care. 1998; 3(4):169-78. DOI: 10.3109/13625189809167250

8. López-De Castro F, Lombardía-Prieto J. Novedades en anticoncepción hormonal. Inf Ter Sist Nac Salud. 2005; 29(3): 57-68.

9. Botella-Llusiá J, Clavero-Núñez JA. Contracepción. Tratado de Ginecología. Editorial Científico-Técnica. La Habana. Edición 14. 1999; 851-73.

10. FNUAP. El Estado de la Población Mundial. PNUD. 2001; 3-7.

11. Espitia-De La Hoz FJ. Prevalencia de disfunción sexual en mujeres del Eje Cafetero. Rev Cienc Biomed 2016; 7(1):1523.

12. Blümel JE, Binfa L, Cataldo $P$, Carrasco A, Izaguirre H, Sarrá $\mathrm{S}$. Índice de función sexual femenina: un test para evaluar la sexualidad de la mujer. Rev Chil Obstet Ginecol. 2004; 69(2):118-25.

13. Espitia-De La Hoz FJ. Prevalence and characterisation of sexual dysfunctions in women, in 12 colombian cities, 20092016. Rev Colomb Obstet Ginecol. 2018; 69 (1):9-21. DOI: http://dx.doi.org /10.18597/rcog.3035

14. Eliason S, Awoonor-Williams JK, Eliason C, Novignon J, Nonvignon J, Aikins M. Determinants of modern family planning use among women of reproductive age in the Nkwanta district of Ghana: a case-control study. Reprod Health. 2014; 11(1):65. doi: 10.1186/1742-4755-11-65

15. Apanga PA, Adam MA. Factors influencing the uptake of family planning services in the Talensi District, Ghana. Pan Afr Med J. 2015; 20:10. doi: 10.11604/pamj.2015.20.10.5301

16. Fagan T, Dutta A, Rosen J, Olivetti A and Klein K. Family planning in the context of Latin America's universal health coverage agenda. Global Health: Science and Practice. 2017; 5(3):382-98. DOI: https://doi.org/10.9745/GHSP-D-17-00057

17. Ribeiro CCM, Shimo AKK, Lopes MHBM, Lamas JLT. Effects of different hormonal contraceptives in women's blood pressure values. Rev Bras Enferm [Internet]. 2018;71(Suppl 3):1453-9. DOI: http://dx.doi.org/10.1590/0034-7167-20170317

United Nations (UN), Department of Economic and Social Affairs, Population Division. Trends in contraceptive use worldwide 2015. New York: UN; 2015. http://www.un.org/en/development/desa/population/publications/pdf/family/trendsContraceptiveUse2015Report.pdf. Accessed february 02, 2020.

18. VI ENCUESTA Grupo DAPHNE 2009. Disponible en: http:// www.equipodaphne.es/ (acceso 02 de febrero de 2020).

19. OMS/UNFPA (Organización Mundial de la Salud/Fondo de Población de las Naciones Unidas). Seguimiento a nivel nacional de los logros en el acceso universal a la salud reproductiva: Consideraciones conceptuales y prácticas e indicadores relacionados. Ginebra: OMS; 2009.

20. Cuervo-Vergara SA, Garrido-Gutiérrez JD, Vélez-Álvarez GA, Zuleta-Tobón JJ. Asociación entre conocimientos de anticoncepción y embarazo no planeado. Estudio de corte transversal. Rev. Colomb. Obstet. Ginecol. 2015; 66(3):1607. DOI: https://doi.org/10.18597/rcog.21

21. Gebremedhin M, Tesfaye G, Belachew A, Desta D. Factors influencing modern contraceptive method preference among women of reproductive age in central zone of Tigray Region, 
Northern Ethiopia. International Journal of Healthcare. 2012; 2(1):82-9. doi:10.5430/ijh.v2n1p82

22. Tengia-Kessy A, Rwabudongo N. Utilization of modern family planning methods among women of reproductive age in a rural setting: the case of Shinyanga rural district, Tanzania. East African Journal of Public Heath 2006; 3:26-30.

23. García Sevillano L, Lobato Moreno S. Dispensación de anticonceptivos hormonales en una farmacia comunitaria de Valladolid. Ars Pharm. 2013; 54(2): 12-9.

24. Petruta A, García-Jiménez E, Martínez F, Moreno A. Grado de conocimiento sobre el uso de anticonceptivos hormonales en una farmacia comunitaria en Zaragoza. Ars Pharm. 2009; 50(1):1-7.

25. Halpern V, Lopez LM, Grimes DA, Stockton LL, Gallo MF. Strategies to improve adherence and acceptability of hormonal methods of contraception. Cochrane Database Syst Rev. 2011;10:CD004317.

26. Grossman Barr N. Managing adverse effects of hormonal contraceptives. Am Fam Physician. 2010; 82(12):1499-506.

27. Loder EW, Buse DC, Golub JR. Headache and combination estrogen-progestin oral contraceptives: integrating evidence, guidelines, and clinical practice. Am J Obstet Gynecol. 2005; 45(3):224-31. DOI: 10.1111/j.1526-4610.2005.05049.x

28. Sabatini R, Cagiano R. Comparison profiles of cycle control, side effects and sexual satisfaction of three hormonal contraceptives. Contraception. 2006; 74(3):220-3. DOI: 10.1016 / j.contraception.2006.03.022
29. Espitia-De La Hoz FJ. O-08 Alteration of the Sexual Response Cycle in Women Using Combined Oral Contraceptives. The Journal of Sexual Medicine. 2017; 14(12), e374. doi:10.1016/j.jsxm.2017.10.017

30. Schaffir J. Hormonal contraception and sexual desire: a critical review. J Sex Marital Ther. 2006; 32(4):305-14. DOI: 10.1080/00926230600666311

31. Berenson AB, Odom SD, Breitkopf CR, Rahman M. Physiologic and psychologic symptoms associated with use of injectable contraception and 20 microg oral contraceptive pills. Am J Obstet Gynecol. 2008; 199(4):351.e1-351.e12.

32. Szarewski A, Mansour D, Shulman LP. 50 years of "The Pill": celebrating a golden anniversary. J Fam Plann Reprod Health Care. 2010; 36(4):231-8.

33. Espitia-De La Hoz FJ. Fisiopatología del trastorno del deseo sexual en el climaterio. Rev. Méd. Risaralda 2018; 24(1):5860. DOI: http://dx.doi.org/10.22517/25395203.14521

\section{Correspondencia:}

Franklin José Espitia De La Hoz.

Dirección: Carrera 12 \# 0 - 75, Consultorio 508 Clínica del Café Armenia, Quindío, Colombia

Correo: espitiafranklin@hotmail.com

Teléfono: 3127436696 